\section{Pontes de miocárdio em cães. II. Topologia}

\author{
Myocardial bridges in dogs. II. Topology
}

\author{
Ricardo Coutinho do AMARAL'; Pedro Primo BOMBONATO² ${ }^{2}$ Frederico Ozanan \\ Carneiro e SILVA ${ }^{3}$; Renato Souto SEVERINO ${ }^{3}$; Sérgio Salazar DRUMMOND ${ }^{3}$; \\ Duvaldo EURIDES ${ }^{3}$
}

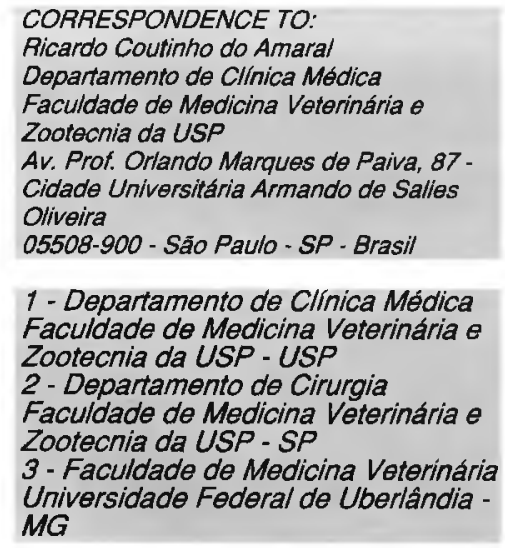

\title{
RESUMO
}

Estudou-se em 134 corações de cães de diferentes raças a localização das pontes de miocárdio, mediante dissecação das artérias coronárias injetadas com solução de gelatina ou Neoprene Látex. Verificou-se que as pontes de miocárdio ocorrem em $90,43 \%$ nos ramos da a. coronária esquerda e em 9,57\% nos ramos da a. coronária direita. Sua posição foi assinalada em $37,23 \%$ na porção dorsal do ventrículo, em $28,72 \%$ na média, em $21,28 \%$ na ventral, em $7,45 \%$ no ápice e simultaneamente nas porções dorsal e média em $3,19 \%$ e nas porções média e ventral em $2,13 \%$. Não foram notadas diferenças estatisticamente significativas em relação ao sexo.

\section{UNITERMOS: Anatomia; Cães; Coração; Miocárdio; Ponte de artéria coronária.}

\section{INTRODUÇÃO}

$\mathrm{N}$ estas últimas décadas talvez seja o coração o órgão que tenha merecido maior atenção dos pesquisadores, que o têm estudado sobre diferentes aspectos, quer da sua morfologia microscópica, como macroscópica, quer também de sua estrutura funcional.

No estudo da sua morfologia tem-se procurado encontrar respostas ou subsídios para a interpretação das diferentes patologias, que vêm sendo diagnosticadas com o auxílio de exames complementares, que se tornam cada vez mais minuciosos e eficientes.

O comportamento da tunelizaçāo das artérias do coração em sua musculatura tem sido objeto de vários estudos, como o de

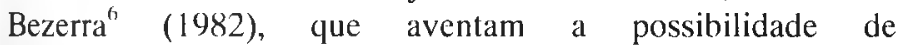
responsabilizá-la, na espécie humana, como um dos fatores que contribuem para o desencadeamento de processos anginosos (Décourt et al. $\left.{ }^{9}, 1980\right)$ ), a despeito de controversos dados ou conhecimentos relativos ao assunto. Este fato fica ainda mais evidente quando observamos a literatura concernente aos animais, em que notamos, nos parcos relatos, a tendência de generalização dos conhecimentos de uma espécie para outras.

$\mathrm{Na}$ expectativa de fornecer novos conhecimentos sobre a morfologia do coração, particularmente no referente à anatomia animal comparada, este trabalho tem como objetivo estudar a localização, mais especificamente, o lado, a posição e a vasculotopia das pontes de miocárdio em cães de raças definidas, contribuindo com dados sistemáticos para a literatura relativa ao objeto de estudo.

\section{MATERIAL E MÉTODO}

Para a realização desta pesquisa utilizamos 134 corações de cães com raças definidas, 80 machos e 54 fêmeas, jovens e adultos, provenientes de várias clínicas veterinárias da cidade de São Paulo e do Hospital Veterinário da Faculdade de Medicina Veterinária e Zootecnia da Universidade de São Paulo.

Após o óbito dos animais, sem história de patologia cardíaca, os coraçóes foram isolados e tiveram scus átrios c ventrículos esvaziados por mcio de massagens c lavagens sucessivas. A seguir as artérias coronárias foram injetadas, separadamente, com solução de gelatina a $10 \%(\mathrm{p} / \mathrm{v})$ corada pelo "cinabrio" (Hgb)* ou com solução de Neoprene Látex "450"** tingida com corante específico c, após fixação cm solução aquosa de formol a $10 \%$, por no mínimo 48 horas, realizamos a dissecação das artérias coronárias e scus principais ramos ventriculares.

Logo após a dissecação, utilizando-nos de um paquímetro, mensuramos a largura das pontes de miocárdio e a altura dos ventrículos correspondentes.

Os resultados foram descritos, adotando-se a nomenclatura (dos vasos coronarianos e seus ramos) proposta por Lücke ${ }^{16}$ (1955) com reparos de Habermehl ${ }^{13}$ (1959), e na análise 
AMARAL, R.C.; BOMBONATO, P.P.; SILVA, F.O.C.; SEVERINO, R.S.; DRUMMOND, S.S.; EURIDES, D. Pontes de miocárdio em cães. I1. Topologia. Braz. J. vet. Res. anim. Scl. São Paulo, v.33, n.4, p.207-213, 1996.

\section{Tabela 1}

Número de pontes de milocárdlo em animais da espécie canina, segundo a raça, lado de ocorrêncla e sexo. São Paulo, 1989.

\begin{tabular}{l|c|c|c|c|c}
\multicolumn{1}{c}{ Raça } & \multicolumn{2}{|c|}{ Lado direito } & \multicolumn{2}{c|}{ Lado esquerdo } & Total \\
\cline { 2 - 5 } & fêmea & macho & fêmea & macho & \\
\hline Basset Hound & - & - & - & 1 & 1 \\
Borzoi & - & - & - & - & - \\
Boxer & - & 4 & 8 & 6 & 18 \\
Chow Chow & - & - & - & 1 & 1 \\
Cocker Spaniel & - & - & - & 3 & 3 \\
Collie & - & - & 2 & 5 & 7 \\
Dálmata & - & 1 & 11 & - & 12 \\
Dachshund & - & - & - & 4 & 4 \\
Doberman & - & - & 1 & - & 1 \\
Dogue Alemão & - & - & 1 & 1 & 2 \\
Fila Brasileiro & - & - & 4 & 3 & 7 \\
Fox Terrler & - & - & - & - & - \\
Greyhound & - & - & - & 1 & 1 \\
Husky Siberiano & - & - & - & 3 & 3 \\
Pointer & - & - & - & 2 & 2 \\
Poodle & - & 1 & 5 & 17 & 23 \\
São Bernardo & - & 1 & 2 & 1 & 4 \\
Schnauzer & - & - & 2 & - & 2 \\
Setter Irlandès & - & - & 2 & - & 2 \\
Weimaraner & - & - & - & 1 & 1 \\
\hline Total & - & 7 & 38 & 49 & 94 \\
\hline
\end{tabular}

\section{B - POSIÇÃO}

Para a descrição da posição da ponte de miocárdio, tomamos a altura do ventrículo compreendida da margem dorsal do sulco coronário, na face esquerda junto à emergência dos ramos circunflexo e interventricular paraconal e na face direita junto à origem do ramo interventricular subsinuoso, até a extremidade do ápice cardíaco e dividimo-la, eqüitativamente, em porção dorsal, média, ventral e apical, esta última tomando como ponto de reparo dorsal a curvatura do sulco interventricular esquerdo da face esquerda para a direita, em função do vértice cardíaco acessório, de sorte que as pontes foram identificadas mais freqüentemente situadas na porção dorsal - $35(37,23 \%)$ vezes, $20(21,28 \%) \mathrm{em}$ machos e $15(15,95 \%)$ em fêmeas; na porção média - 27 $(28,72 \%)$ vezes, $16(17,02 \%)$ em machos e $11(11,70 \%)$ em fêmeas; na porção ventral - $20(21,28 \%)$ vezes, 14 $(14,90 \%)$ em machos e $6(6,38 \%)$ em fêmeas; no ápice $7(7,45 \%)$ vezes, $3(3,19 \%)$ vezes em machos e $4(4,25 \%)$ em fêmeas; na porção dorsal e média - 3 (3,19\%) vezes, $2(2,13 \%)$ em machos e $1(1,06 \%)$ em fêmeas, e na porção média e ventral - $2(2,13 \%)$ vezes, exclusivamente em fêmeas.

A posição das pontes de miocárdio variou $\mathrm{em}$ decorrência da raça do animal, com distribuição própria. conforme assinalado na Tab. 2.

As pontes ocorridas em ramos da artéria coronária esquerda, a saber, $85(90,43 \%)$ vezes, $46(48,94 \%)$ em machos e $39(41,49 \%)$ em fêmeas, foram vistas mais estatística utilizamos os testes de $\mathrm{X}^{2}$ e de suas proporções com aproximaçāo ao nível de significância de $5 \%$ e dos testes de correlação de Spearman, segundo Snedecor; $\operatorname{Cochran}^{19}$ (1967), com significância de $1,0 \%$.

* Carlo Erba

\section{RESULTADOS}

\section{A - LADO}

Em cães de raça notamos a ocorrência de 94 pontes de miocárdio de largura variada, em $45,52 \%$ das peças examinadas e distribuídas em 55 pontes $(58,51 \%)$ em machos e 39 pontes $(41,49 \%)$ em fêmeas. Esta entidade ocorreu com maior frequiência na face esquerda ou auricular do coração, mais especificamente $87(92,55 \%)$ pontes, sendo $48(51,06 \%)$ em machos e $39(41,49 \%)$ em fêmeas. Já na face direita ou atrial, pudemos identificar $7(7,44 \%)$ pontes, exclusivamente em machos.

Quando analisamos o material, levando em consideração a raça, notamos distribuição própria, como descrita na Tab. 1. freqüentemente na porção dorsal - $33(35,11 \%)$ vezes, 18 $(19,15 \%)$ em machos e $15(15,96 \%)$ em fêmeas; na porção média - $25(26,59 \%)$ vezes, $14(14,89 \%)$ em machos e 11 $(11,70 \%)$ em fêmeas; na porção ventral - $16(17,03 \%)$ vezes, $10(10,64 \%)$ em machos e $6(6,39 \%)$ em fêmeas; na porção apical - $6(6,38 \%)$ vezes, $2(2,13 \%)$ em machos e $4(4,25 \%)$ em fêmeas; na porção dorsal e média - $3(3,19 \%)$ vezes, 2 $(2,13 \%)$ em machos e $1(1,06 \%)$ em fêmeas; na porção média e ventral - $2(2,13)$ vezes, somente em fêmeas.

As pontes observadas em ramos da artéria coronária direita, a saber, $9(9,57 \%)$ vezes, ocorridas exclusivamente em machos, foram assinaladas mais freqüentemente na porçāo ventral - $4(4,25 \%)$ vezes; na porção dorsal - $2(2,13 \%)$ vezes; na porção média - $2(2,13 \%)$ vezes; na porção apical - 1 $(1,06 \%)$ vez.

\section{C - VASCULOTOPIA}

As pontes de miocárdio foram identificadas em ramos da artéria coronária esquerda em $85(90,43 \%)$ vezes, sendo 46 $(48,49 \%)$ em machos e $39(41,49 \%)$ em fêmeas e em ramos da artéria coronária direita por $9(9,57 \%)$ vezes, exclusivamente em machos. 
AMARAL. R.C.; BOMizONATO, P.P.; SILVA, F.O.C.; SEVERINO, R.S.; DRUMMOND, S.S.; EURIDES, D. Pontes de miocárdio em cães. II. Topologia. Braz. J. vet. Res. anim. Sci. Säo Paulo, v.33, n.4, p.207-213, 1996.

Tabela 2

Animais da espécie canina, segundo raça, largura (em cm) e posição das pontes de miocárdio. São Paulo, 1989.

\begin{tabular}{|c|c|c|c|c|c|c|}
\hline \multirow[b]{2}{*}{ RAÇA } & \multicolumn{6}{|c|}{ POSIÇĀO } \\
\hline & Dorsal & Média & Ventral & Apice & Dorsal - média & Média - ventral \\
\hline Basset Hound & 0,70 & - & - & - & $=$ & - \\
\hline Borzoi & - & - & - & - & - & - \\
\hline Boxer & 1,10 & 1,00 & 1,50 & 0,60 & 1,20 & 1,30 \\
\hline Boxer & 0,20 & 0,80 & 0,40 & 0,60 & - & - \\
\hline Boxer & 0,90 & - & 0,50 & 0,80 & - & - \\
\hline Boxer & 0,10 & - & - & 0,50 & - & - \\
\hline Boxer & 1,20 & - & - & - & - & - \\
\hline Boxer & 0,70 & - & - & - & - & - \\
\hline Boxer & 0,10 & - & - & - & - & - \\
\hline Chow Chow & - & - & 1,90 & - & - & - \\
\hline Cocker Spaniel & 1,00 & 0,60 & - & - & 1,90 & $\cdot$ \\
\hline Collie & 1,40 & 1,20 & 1,20 & - & 2,00 & - \\
\hline Collie & 1,20 & - & 0,50 & - & - & - \\
\hline Collie & 0,40 & - & - & $-j$ & - & - \\
\hline Dálmata & 1,40 & 1,30 & 2,30 & 0,10 & - & - \\
\hline Dálmata & 0,60 & 0,40 & 1,60 & - & - & - \\
\hline Dálmata & 0,90 & 0,30 & - & - & - & - \\
\hline Dálmata & - & 1,50 & - & - & - & - \\
\hline Dálmata & - & 2,30 & - & - & - & - \\
\hline Dálmata & - & 0,40 & - & - & - & - \\
\hline Dachshund & 1,40 & 0,40 & - & - & - & - \\
\hline Dachshund & 0,60 & - & - & - & - & - \\
\hline Dachshund & 0,40 & - & - & - & - & - \\
\hline Doberman & 0,40 & - & - & - & - & - \\
\hline Dogue Alemāo & - & - & 0,40 & 1,20 & - & - \\
\hline Fila Brasileiro & 0,20 & 0,80 & 1,30 & - & - & - \\
\hline Fila Brasileiro & 0,30 & - & 1,00 & - & - & 1,70 \\
\hline Fila Brasileiro & 1,80 & - & - & - & - & - \\
\hline Fox Terrier & - & - & - & - & - & - \\
\hline Greyhound & - & - & 0,40 & - & - & - \\
\hline Husky Siberiano & 1,00 & 0,90 & - & - & - & - \\
\hline Husky Siberiano & - & 0,60 & - & - & - & - \\
\hline Pointer & - & 0,30 & 1,00 & - & - & - \\
\hline Poodle & 0,90 & 1,00 & 1,10 & - & - & - \\
\hline Poodle & 0,30 & 0,70 & 2,00 & - & - & - \\
\hline Poodle & 1,00 & 0,30 & 0,20 & - & - & - \\
\hline Poodle & 1,90 & 0,20 & - & - & - & - \\
\hline Poodle & 0,60 & 1,20 & - & - & - & - \\
\hline Poodle & 0,40 & 0,10 & - & - & - & $\cdot$ \\
\hline Poodle & 1,60 & 0,30 & - & - & - & - \\
\hline Poodle & 1,20 & 0,50 & - & - & - & - \\
\hline Poodle & 1,10 & 1,30 & - & - & - & - \\
\hline Poodle & 0,30 & 0,90 & - & - & - & - \\
\hline São Bernardo & 0,60 & 0,90 & 2,00 & 0,90 & - & $\cdot$ \\
\hline Schnauzer & 1,50 & - & 0,40 & - & - & - \\
\hline Setter Irlandês & 0,30 & 0,60 & - & - & - & - \\
\hline Weimaraner & - & - & 0,60 & - & - & - \\
\hline Valores médios & 0,82 & 0,70 & 1,68 & 0,67 & 1,60 & 1,50 \\
\hline Valores mínimos & 0,10 & 0,10 & 0,10 & 0,50 & 1,20 & 1,30 \\
\hline Valores máximos & 1,90 & 2,30 & 2,00 & 0,90 & 2,00 & 1,70 \\
\hline
\end{tabular}


AMARAl, R.C.; BOMbONATO, P.P.; SILVA, F.O.C; SEVERINO, R.S.; DRUMMOND, S.S.; EuRIDES, D. Pontes de miocárdio em cães. II. Topologii. Braz. J. vet. Res. anim. Sci. São Paulo, v..33, n.4, p.207-213, 1996.

Os vasos arteriais que mais freqüentemente apresentaram pontes de miocárdio foram:

- ramo interventricular paraconal - $62(65,96 \%)$ vezes, 33 $(35,11 \%)$ cm machos e $29(30,85 \%)$ em fêmeas;

- ramos distal - $5(5,32 \%)$ vezes, $2(2,13 \%)$ em machos e 3 $(3,19 \%)$ em fêmeas;

- ramo interventricular subsinuoso quando continuação do ramo circunflexo esquerdo - $4(4,25 \%)$ vezes, apenas em machos;

- ramo da margem ventricular esquerda - $3(3,19 \%)$ vezes, 1 $(1,06 \%)$ em macho e $2(2,13 \%)$ em fêmeas;

- ramo ventricular direito - $3(3,19 \%)$ vezes, $2(2,13 \%) \mathrm{em}$ machos e $1(1,06 \%)$ em fêmea;

- ramo proximal - $2(2,13 \%)$ vezes, $1(1,06 \%)$ em macho e $1(1,06 \%)$ em fêmea;

- ramo ventricular esquerdo proximal - $2(2,13 \%)$ vezes, apenas em machos;

- ramo ventricular esquerdo distal - $2(2,13 \%)$ vezes, apenas cm fêmeas;
- colateral ventricular esquerdo do ramo interventricular subsinuoso - $2(2,13 \%)$ vezes, $1(1,06 \%)$ em macho e 1 $(1,06 \%)$ em fêmea;

- colateral do ramo ventricular direito distal - $2(2,13 \%)$ vezes, apenas em machos;

- ramo ventricular direito proximal - $1(1,06 \%) \mathrm{vez}, \mathrm{em}$ macho;

- ramo ventricular direito distal - $1(1,06 \%)$ vez, em macho; - ramo adiposo - 1 (1,06\%) vez, cm macho.

A vasculotopia das pontes de miocárdio variou em função da raça do animal, com distribuição própria, conforme assinalado na Tab. 3 .

\section{D - ASPECTOS DA ANÁLISE ESTATÍSTICA}

Ao utilizarmos os testes de $\mathrm{X}^{2}$ e de 2 proporções com aproximação normal no nível de significância de $5 \%$, pudemos verificar que, quando analisamos o lado de ocorrência das

Tabela 3

Número de pontes de miocárdio em animais da espécie canina, segundo raça, vasculotopia e sexo. São Paulo, 1989.

\begin{tabular}{|c|c|c|c|c|c|c|c|c|c|c|c|c|c|c|c|c|c|c|c|c|c|c|c|c|c|c|c|c|c|}
\hline \multirow[t]{3}{*}{ RAÇA } & \multicolumn{28}{|c|}{ VASO } & \multirow[t]{3}{*}{ TOTAL } \\
\hline & \multicolumn{2}{|c|}{ A } & \multicolumn{2}{|c|}{$\mathrm{B}$} & \multicolumn{2}{|c|}{ C } & \multicolumn{2}{|c|}{$\mathrm{D}$} & \multicolumn{2}{|c|}{$\mathrm{E}$} & \multicolumn{2}{|c|}{$F$} & \multicolumn{2}{|c|}{$G$} & \multicolumn{2}{|c|}{$\mathrm{H}$} & \multicolumn{2}{|c|}{1} & \multicolumn{2}{|c|}{$J$} & \multicolumn{2}{|c|}{$\bar{L}$} & \multicolumn{2}{|c|}{$\mathrm{M}$} & \multicolumn{2}{|c|}{$\mathrm{N}$} & \multicolumn{2}{|c|}{0} & \\
\hline & $\sigma$ & 9 & $\sigma$ & 9 & $\sigma$ & ? & $\sigma$ & 9 & $\sigma$ & 9 & $\sigma$ & 9 & $\sigma$ & 9 & $\sigma$ & 9 & $\sigma$ & q & $\sigma$ & 9 & $\sigma$ & 9 & $\sigma$ & 9 & $\sigma$ & 9 & $\sigma$ & 9 & \\
\hline Basset Hound & - & 1 & & & - & & & & & & - & & & & & & - & & & & - & - & - & - & - & - & - & - & 1 \\
\hline Borzoi & - & - & & & - & & & - & - & & - & - & 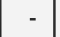 & - & & - & - & & - & & - & - & - & - & - & - & - & - & - \\
\hline Boxer & 4 & 5 & - & 1 & 1 & - & - & - & - & 1 & 1 & - & - & - & - & - & - & & - & 1 & 2 & - & - & - & 1 & - & 1 & - & 18 \\
\hline Chow Chow & 1 & - & - & - & - & - & - & - & - & - & - & - & - & - & - & - & - & - & - & - & - & - & - & - & - & - & - & - & 1 \\
\hline Cocker Spaniel & 1 & - & 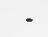 & - & - & - & - & - & - & - & - & - & 1 & - & - & - & - & - & 1 & - & - & - & - & - & - & - & - & - & 3 \\
\hline Collie & 2 & 1 & 1 & - & 1 & - & - & - & - & 1 & 1 & - & - & - & - & - & - & - & - & - & - & - & - & - & - & - & - & - & 7 \\
\hline Dálmata & - & 8 & - & 1 & - & - & 1 & - & - & - & - & - & - & - & - & - & 2 & - & - & - & - & - & -1 & - & - & - & - & - & 12 \\
\hline Dachshund & 4 & - & - & - & - & - & - & - & - & - & - & 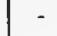 & - & - & -1 & - & - & - & 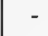 & - & 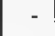 & - & - & 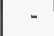 & - & - & - & 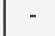 & 4 \\
\hline Doberman & 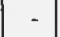 & 1 & - & - & - & - & - & 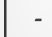 & - & - & - & - & - & - & - & - & - & - & - & - & - & - & - & - & - & - & - & - & 1 \\
\hline Dogue Alemão & 1 & 1 & - & - & - & - & - & 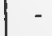 & - & - & - & - & - & 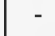 & - & - & - & - & - & - & - & - & - & - & - & - & - & - & 2 \\
\hline Fila Brasileiro & 3 & 1 & - & 1 & - & - & - & - & - & - & - & 1 & - & 1 & - & - & - & - & . & - & - & - & - & - & - & - & - & - & 7 \\
\hline Fox Terrier & - & - & - & - & - & - & - & - & - & - & - & - & - & - & -1 & - & - & - & - & - & - & - & -1 & 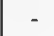 & - & - & - & - & - \\
\hline Greyhound & - & - & 1 & - & - & - & - & - & - & - & - & - & - & - & - & - & - & - & - & - & - & . & - & 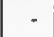 & - & - & - & - & 1 \\
\hline Husky Siberiano & 3 & - & - & - & - & - & - & - & - & - & - & - & -1 & - & - & - & - & - & 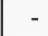 & 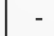 & 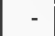 & - & - & - & - & - & - & - & 3 \\
\hline Pointer & & - & - & - & - & - & 2 & - & - & - & - & - & - & - & - & - & - & - & - & - & - & - & - & - & - & - & - & - & 2 \\
\hline Poodle & 12 & 5 & - & - & 2 & - & - & - & 1 & - & - & - & - & - & 2 & - & . & - & - & - & - & - & 1 & - & - & - & - & - & 23 \\
\hline São Bernardo & 1 & 2 & - & - & - & . & 1 & - & - & - & - & - & - & - & -1 & - & - & - & - & 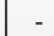 & - & - & - & - & - & - & - & - & 4 \\
\hline Schnauzer & - & 2 & - & - & - & - & - & - & - & 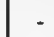 & - & - & - & - & - & - & - & - & - & - & - & - & - & . & . & - & - & - & 2 \\
\hline Setter Irlandês & - & 2 & - & - & - & - & - & - & - & - & - & - & - & - & - & - & - & - & - & - & - & - & - & - & -1 & - & - & - & 2 \\
\hline Weimaraner & 1 & -1 & - & - & - & - & - & - & - & - & - & - & - & - & -1 & - & - & - & - & . & - & - & - & - & -1 & - & - & & 1 \\
\hline SUBTOTAL & 33 & 29 & 2 & 3 & 4 & - & 4 & - & 1 & 2 & 2 & 1 & 1 & 1 & 2 & 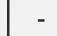 & 2 & - & 1 & 1 & 2 & 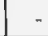 & 1 & - & 1 & & 1 & & 94 \\
\hline TOTAL GERAL & 6 & 32 & 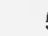 & 5 & & 4 & & 4 & & 3 & & 3 & 2 & 2 & & 2 & & 2 & & 2 & & 2 & 1 & & 1 & 1 & & 1 & 94 \\
\hline
\end{tabular}

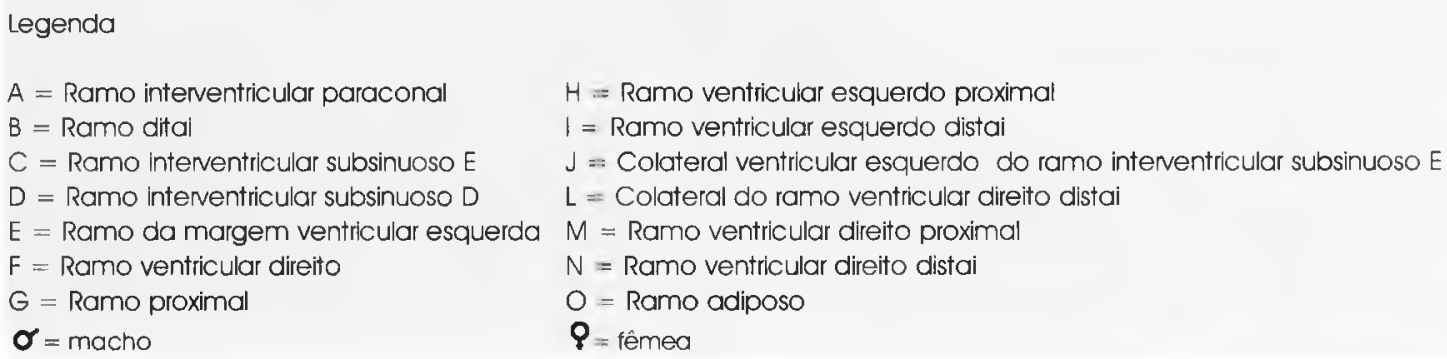


AMARAL, R.C.; BOMBONATO, P.P.; SILVA, F.O.C.; SEVERINO, R.S.; DRUMMOND, S.S.; EURIDES, D. Pontes de miocárdio em cães. II. Topologia. Braz. J. vet.

Res. anim. Sci. Sāo Paulo, v.33, n.4, p.207-213, 1996.

pontes de miocárdio e o sexo, não existem diferenças significantes entre eles.

Ainda, valendo-nos do teste de correlação de Spearman com nível de significância de $1 \%$, encontramos $\mathrm{r}=0,059$ para a correlaçāo entre a posição da ponte e a sua largura, dados estes indicativos de correlaçāo positiva, porém de baixa intensidade, mostrando-nos que após traçada a reta correspondente, não existe indicação de que na decorrência da largura da ponte ela se localizará preferencialmente numa ou noutra posição (Fig.1)

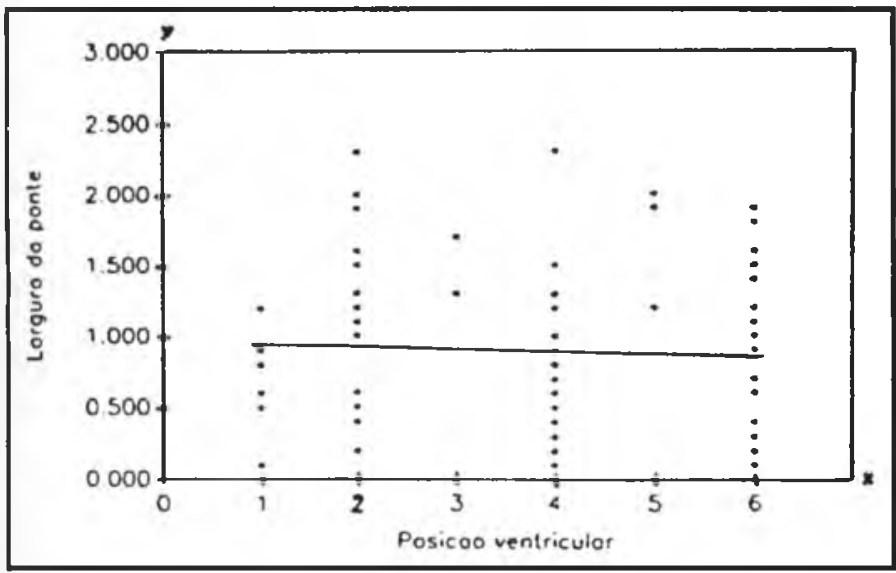

Figura 1

Gráfico representativo da distribuição da largura das pontes de miocárdio $(y) \mathrm{em} \mathrm{cm}$ em relação a sua posição nos ventrículos $(\mathrm{X}) \mathrm{em} \mathrm{cm}$.

$\begin{array}{ll}\text { Legenda } & \\ \text { i-Ápice } & \text { 4-Média } \\ \text { 2-Ventral } & \text { 5-Dorsal Média } \\ \text { 3-Média Ventral } & \text { 6-Dorsal }\end{array}$

\section{DISCUSSĀO}

Ao analisarmos a literatura podemos notar que os autores clássicos, como, por exemplo, Bossi et al. ${ }^{7}$ (s.d.); Bruni; Zimmerl $^{\text {}}$ (1947); Hoffmann ${ }^{15}$ (1975); Ellenberger; Baum ${ }^{\text {II }}$ (1977); Evans; Christensen ${ }^{11}$ (1979): Getty ${ }^{12}$ (1981) e Nickel et al. ${ }^{17}$ (1981), não atentam para a presença de pontes de miocárdio em vasos coronarianos, procurando tão-somente descrever a origem e distribuiçāo desses vasos, mesmo aqueles que tratam especificamente da anatomia do cão. Isto nos leva à confrontaçáo dos nossos achados somente com aqueles oriundos da literatura especializada.

Assim, encontramos Berg ${ }^{2}$ (1963), que, apesar de em seu trabalho realizado em suínos sugerir que os achados seriam semelhantes para os cães, sua descrição se detém apenas na freqüência, sem considerar o vaso, a posição ou a face do coração em que ocorreria a referida entidade, variáveis estudadas em nosso trabalho, o que, a nosso ver, limita a possibilidade de generalização assinalada pelo autor.

Em outro trabalho, Berg.4 (1964) apresenta dados que também diferem em muito dos nossos, ou seja, de 37 corações de câes examinados, encontra somente 1 ponte de miocárdio na artéria coronária esquerda, em seu ramo interventricular paraconal; já em nosso material tivemos a oportunidade de identificar 94 pontes de miocárdio, que, apesar de serem mais freqüentemente vistas na artéria coronária esquerda, como Berg observou, também pudemos surpreendê-las na artéria coronária direita.

Haidziselimovic et al. $^{14}$ (1974) relatam a existência de pontes de miocárdio, porém nada descrevem a respeito de sua localização, não permitindo portanto uma confrontação dircta com os nossos resultados. Esses autores porém afirmam que o comportamento coronariano é um problema fillogenélico, representando um estágio intermediário do desenvolvimento. No entanto, esses autores não fornecem subsídios para estas afirmações em suas pesquisas.

Nie; Vincent ${ }^{18}$ (1984), em recente estudo, analisando corações de cāes, encontram pontes de miocárdio localizadas no ramo interventricular paraconal, subsinuoso e seus colaterais, bem como encontram órgãos com múltiplas pontes, coincidindo sobremaneira com nossos resultados. Entretanto, pelo fato de possuirmos este estudo apenas em forma de sumário nos Anais do Seventh European Anatomical Congress, não tivemos acesso a informaçōes mais objetivas no que tange à freqüência em cada vaso ou posição dessas pontes em relação à massa ventricular.

Bertolini et al..$^{5}$ (1994), ao estudarem as pontes de miocárdio em corações de suínos, apontam resultados diferentes daqueles por nós observados em relação à vasculotopia no cão, ou seja, enquanto nós identificamos $65,96 \%$ de pontes situadas sobre o ramo interventricular paraconal, os primeiros indicam $43,75 \%$. Resultados ainda mais discrepantes sāo assinalados em relação às pontes sobre 0 ramo interventricular subsinuoso, sobre o qual observamos $4,25 \%$, contra $37,5 \%$ nos suínos. Entretanto, somos concordes com o relato dos achados estatísticos que demonstram náo existirem diferenças significantes quanto à frequêencia de pontes sobre cada um dos vasos ou em relação ao sexo.

Acreditamos poder debitar as diferenças encontradas entre a topologia das pontes citadas no trabalho de Bertolini et al." (1994) e os nossos resultados às diferenças decorrentes da espécie estudada.

Quanto à origem do ramo interventricular subsinuoso, apesar de não ter sido o objeto maior de nossos estudos, vale notar a coincidência de nossos resultados, nos quais achamos 97,76\% dos casos, com os de Andretto et al.' (1973), que os descrevem em 97,6\% dos casos como continuação direta do ramo circunflexo esquerdo. Já Berg ${ }^{2}$ (1963) afirma ser no cão o ramo interventricular subsinuoso oriundo da artéria coronária esquerda, sem acrescentar a possibilidade por nós verificada, o que, a nosso ver, destaca a importância da descrição da face cardíaca em que ocorrem as pontes, já que em percentual, ainda que reduzido, poderemos ter pontes de miocárdio em ramos da a. coronária esquerda, mas nāo necessariamente na face esquerda.

Em relação à posição das pontes, podemos ressaltar o fato de que elas ocorrem com maior freqüência nas porções mais 
AMARAL, R.C.; BOMBONATO, P.P.; SILVA, F.O.C.; SEVERINO, R.S.; DRUMMOND, S.S.; EURIDES, D. Pontes de miocárdio em cāes. II. Topologia. Braz. J. vet. Res, anim. Sci. Sāo Paulo, v.33, n.4, p.207-213, 1996.

dorsais dos ventrículos, talvez em decorrência das modificações mais acentuadas da angulação das fibras musculares para se prenderem no anel fibroso.

Relativamente às análises estatísticas realizadas em nosso trabalho, acreditamos que vale destacar que, apesar da tendência positiva mostrada, quando correlacionamos a posição da ponte e sua respectiva largura, não há indicação de que as pontes localizadas nas regiões mais dorsais são proporcionalmente mais largas ou mais estreitas do que aquelas situadas em posição apical.

A análise dos resultados ora obtidos, relativamente às diferentes raças estudadas em confronto com a frequiência, largura, posição e vasculotopia, apesar de sua importância morfológica, pode resultar em dados de valor biológico discutível, o que sugere a ampliação e continuação do trabalho agora iniciado.

\section{CONCLUSÕES}

Em consonância com os resultados obtidos, acreditamos serem possíveis as seguintes conclusões:

1) Ocorrência de pontes de miocárdio é observada com maior freqüência nos ramos da artéria coronária esquerda, sem contudo existirem diferenças estatisticamente significativas relativamente ao sexo;

2) Independentemente do vaso, as pontes de miocárdio ocorrem mais freqüentemente na porção dorsal, seguida da média; na ventral, no ápice, ou simultaneamente nas porções dorsal e média; e média e ventral, não havendo diferenças estatisticamente significantes quanto à posição das pontes de miocárdio em relação ao sexo;

3) Nos ramos da artéria coronária esquerda, as pontes são vistas, por ordem, na porção dorsal; na média; na ventral; no ápice; ou simultaneamente nas porções dorsal e média e média e ventral, enquanto nos ramos da artéria coronária direita elas ocorrem, segundo a freqüência, nas porções ventral; dorsal; média; e apical;

4) Nos cães os tratos pontinos, considerando o número total de pontes, ocorrem, por ordem, em segmentos dos seguintes vasos: ramo interventricular paraconal; ramo distal; ramo interventricular subsinuoso da artéria coronária esquerda, ramo interventricular subsinuoso da artéria coronária direita; ramo da margem ventricular esquerda, ramo ventricular esquerda, ramo ventricular direito; ramo proximal; ramo ventricular esquerdo proximal; ramo ventricular esquerdo distal; colateral do ramo ventricular direito distal; colateral ventricular esquerdo do ramo interventricular subsinuoso da artéria coronária esquerda; ramo ventricular direito proximal; ramo ventricular direito distal e ramo adiposo;

5) Nos corações de cães de raça, correlação de baixa intensidade, no nível de $1 \%$, foi assinalada quando comparadas as posições dessas pontes e suas respectivas larguras, sem no entanto existir tendência de proporcionalidade entre os parâmetros.

\section{SUMMARY}

The authors have studied the location of myocardial bridges in 134 hearts of different breed, pure dogs. After injection of Neoprene Latex or gelatine solution, the coronary arteries were dissected. Myocardial bridges (90.43\%) were observed in branches of the left coronary arteries, and $9.57 \%$ in branches of the right coronary arteries. The bridges' locations were: $37.23 \%$ in the dorsal portion; $28.72 \%$ in the medium portion; $21.28 \%$ in the ventral portion; $7.45 \%$ in the vertex portion; $3.19 \%$ simultaneously in the dorsal and medium portions and $2.13 \%$ simultaneously in the medium and ventral portions. Statistical differences were not observed between males and females.

UNITERMS: Anatomy; Dogs; Heart; Myocardium.

\section{REFERENCES}

1-ANDRETTO, R.; BORELLI, V.; FERNANDES FILHO, A. Sobre a origem do ramus descendens subsinuosus em cães. Revista da Faculdade de Medicina Veterinária e Zootecnia da Universidade de São Paulo, v.10, n.1, p.510,1973 .

2-BERG, R. Uber das Auftreten von Myocardbrücken über den Koronarfegässen beim Schwein (Sus scrofa domesticus). Anatomischer Anzeizer, v.112, n.1, p.25-31, 1963

3-BERG, R. Beitrag zur Phylogenese des Verhaltens der Koronararterien zum Myokard beim Hausschwein (Sus scrofa domesticus). Anatomischer Anzeizer, v.115, n.2, p.184-92, 1964.

4-BERG, R. Uber den Entwicklungsgrad des Koronargefass - musters ä beim Hausschwein (Sus scrofa domesticus). Anatomischer Anzeizer, v.115, n.2 p.193-204, 1964.
5-BERTOLLINI, S.M.G.; PRATES, N.E.V.B.; MIRANDA NETO, M.H. Estudo macroscópico e estatístico das pontes de miocárdio sobre as artérias coronárias de suínos. Revista UNIMAR, v.16, p.383-93, 1994

6-BEZERRA, A.J.C. Contribuiçāo para o conhecimento das pontes de miocárdio. Sāo Paulo, 1982. 169p. Dissertação (Mestrado) - Escola Paulista de Medicina.

7-BOSS1, V.; CARADONNA, G.B.; SPAMPANI, G.; VARALDI, L.; ZIMMERL. U. Trattato di anatomia veterinaria. Milano, Francesco Vallardi, s.d. v.2, p.344.

8-BRUNI, A.C.; ZIMMERL, U. Anatomia degli animali domestici. Milano, Francesco Vallardi, 1947 v.2, p.290-304.

9-DÉCOURT, L.V.; CARVALHO, V.B.; MARTINEZ, J.R.M. Ponte miocárdica. Uma entidade controvertida. Revista do Hospital das Clínicas da Faculdade de Medicina da Universidade de Sāo Paulo, v.35, n.3, p. I57-60, 1980

10-ELLENBERGER, W.; BAUM, H. Handbuch der vergleichenden Anatomie der Haustiere. 18. auf. Berlin, Springer, 1977. p.610-26 
AMARAL, R.C.; BOMBONATO, P.P.; SILVA, F.O.C.; SEVERINO, R.S.; DRUMMOND, S.S.; EURIDES, D. Pontes de miocárdio em cães. II. Topologia. Braz. J. vet. Res. anim. Sci. São Paulo, v.33, n.4, p.207-213, 1996.

11-EVANS, H.E.; CRISTENSEN, G.C. Miller's anatomy of the dog. 2.ed. Philadelphia, W.B. Saunders, 1979. p.632-51.

12-GETTY, R. Sisson and Grossmann's the anatomy of the domestic animals 5.ed. Philadelphia, W.B. Saunders, 1981. v.1, p.14-7, 153-62; v.2, 1497-501.

13-HABERMEHL, K.H. Blutgefässversorgung des Katzenherzens. Zentralblat fur Veterinarmedizin, A, v.6, p.655-80, 1959.

14-HADZISELIMOVIC, H.; SECEROV, D.; GMAZ-NIKULIN, E. Comparative anatomical investigations on coronary arteries in wild and domestic animals. Acta Anatomica, v.90, n.1, p.16-35, 1974.

15-HOFFMANN, V. Die Blutgefässversorgung des Pferdeherzens, zugleich auch eine vergleichende Betrachtung der Topographie der herzeigenen Blutgefásse der Haussāuger Fleischfresser, Schwein und Wiederkăuer. Anatomischer Anzeizer, v.137, n.1, p.79-109, 1975.

16-LUCKE, R. Blutgefässversorgung des Hundeherzens. Hannover, 1955. 69p. (Inaugural Dissertation) Tierärztliche Hochschule.

17-NICKEL, R.; SCHUMMER, A.; SEIFERLE, E. The anatomy of the domestic animals. Berlin, Paul Parey, 1981. v.3, p.15-49.

18-NIE, C.J. VAN; VINCENT, J.G. Myocardial bridges on the coronary arteries in animals. Acta Anatomica, v.120, n.1, p.53, 1984.

19-SNEDECOR, G.W.; COCHRAN, W.G. Statistical methods. 6.ed. Ames, The Iowa State University Press, 1967. p.135-7, 193-5. 